\title{
The Role of sUPAR and SAPS II on the Ventilator-Associated Pneumonia
}

\section{Havva Alnıkızıl, Mediha Turktan, Ersel Gulec, Feride Karacaer, Dilek Ozcengiz}

Background: Ventilator associated pneumonia (VAP) is one of the serious clinical condition in mechanically ventilated patients. The aim of this study to investigate the effect of the Simplified Acute Physology Score (SAPS) II, soluble urokinase plasminogen activator receptor (sUPAR), C-reactive protein (CRP), procalcitonin(PCT) and lactate values on mortality for VAP.

Materials and Methods: After obtaining the ethics committee aproval, fifty-four patients in intensive care unit (ICU) over 18 years old and diagnosed with VAP were included in this study. SAPS II values were calculated and recorded. Blood samples were collected in order to detect sUPAR, CRP, PCT and lactate values at the first and fifth days. Patients were followed-up during 28 days and recorded as recovered, under follow-up or exitus.

Results and Discussion: The average age of the patients was $57.1 \pm 15.6$ years, length of stay ICU was 21.2 \pm 20.4 days. At the end of 28 day, $59.3 \%$ of patients were exitus, $9.3 \%$ of patients were discharged and $31.5 \%$ of patients were continued the follow-up.

At the fifth day sUPAR values were higher than the first day $(\mathrm{p}=0.028)$ (Table 1). Procalcitonin values at the first day were higher than the fifth day $(\mathrm{p}=0.007)$ (Table 1). There were no significantly differences in lactate and CRP values ( $\mathrm{p}>0.05)$ (Table 1). There were no correlations between sUPAR values at first and fifth days and SAPS II values $(\mathrm{p}=0.211, \mathrm{r}=0.170, \mathrm{p}=0.178, \mathrm{r}=0.190$, respectively). There were no correlations between sUPAR values at first and fifth days and SAPS II predictive mortality percentage $(\mathrm{p}=0.200, \mathrm{r}=0.180, \mathrm{p}=0.169, \mathrm{r}=0.190$, respectively).

In alive patients, CRP and PCT values at the fifth day, SAPS II values and SAPS II predictive mortality percentage were lower than dead patients $(\mathrm{p}=0.017, \mathrm{p}=0.035, \mathrm{p}=0.0001, \mathrm{p}=0.0001$, respectively) (Table 2). In logistic regression model, length of stay ICU, SAPS II predictive mortality percentage, sUPAR, lactate and CRP values at the first day were found as independent risk factors affecting mortality $(\mathrm{p}=0.001, \mathrm{p}=0.003, \mathrm{p}=0.045$, $\mathrm{p}=0.028, \mathrm{p}=0.020$, respectively). A statistically significant cut-off value could not be obtained for sUPAR. When used a value of 45 for SAPS II, $71.9 \%$ sensitivity and $68.2 \%$ specificity were obtained. 
Conclusions: For VAP, sUPAR has no prognostic value but it may be an independent risk factor for mortality. SAPS II is a prognostic factor for these patients and high SAPS II values may be associated with an increased risk of mortality.

Keywords: Ventilator-associated pneumonia, SAPS II, sUPAR, CRP, procalcitonin, lactate

Table 1.Laboratory test values

\begin{tabular}{|l|c|c|c|c|c|c|}
\hline \multirow{2}{*}{} & \multirow{2}{*}{$\mathbf{n}$} & \multicolumn{2}{|c|}{$\mathbf{1}^{\text {th }}$ day } & \multicolumn{2}{c|}{$\mathbf{5}^{\text {th }}$ day } & p \\
\cline { 3 - 7 } & & Mean \pm SD & Med (min-max) & Mean \pm SD & Med (min-max) & $\mathbf{0 , 0 2 8}$ \\
\hline sUPAR & 54 & $7,8 \pm 6,1$ & $5,2(1,5-20,8)$ & $8,5 \pm 6,2$ & $6,6(1-21)$ & 0,763 \\
\hline lactate & 54 & $2,3 \pm 0,9$ & $2,1(1,0-5,2)$ & $2,4 \pm 2,2$ & $2,2(0,3-10,6)$ & 0,183 \\
\hline CRP & 54 & $11,3 \pm 11,1$ & $8,6(0,5-44,8)$ & $9,2 \pm 9,7$ & $6,5(0,3-47,8)$ & 0,007 \\
\hline PCT & 54 & $6,6 \pm 15,4$ & $1,1(0,0-100,0)$ & $3,1 \pm 6,9$ & $0,5(0,0-30,7)$ & $\mathbf{0 , 0 0 7}$ \\
\hline
\end{tabular}

Table 2. The distribution of demographic and clinical variables

\begin{tabular}{|c|c|c|c|c|c|}
\hline & \multicolumn{2}{|c|}{ Alive $(n=22)$} & \multicolumn{2}{|c|}{ Dead $(n=32)$} & \multirow{2}{*}{$\mathbf{p}$} \\
\hline & Mean & Med (Min-Max) & Mean & Med (Min-Max) & \\
\hline Age & 55,6 & $56(23-77)$ & 58,1 & $60(19-80)$ & 0,503 \\
\hline Length of stay ICU & 29,7 & $27(5-143)$ & 15,2 & $15(5-40)$ & 0,025 \\
\hline SUPAR $1^{\text {th }}$ day & 8,6 & $6,1(1,6-20,8)$ & 7,2 & $5(1,5-20,8)$ & 0,526 \\
\hline SUPAR $5^{\text {th }}$ day & 8,7 & $5,5(2-21)$ & 8,4 & $7,1(1-21)$ & 0,764 \\
\hline Laktat $1^{\text {th }}$ day & 2,0 & $1,6(1,3-3,3)$ & 2,5 & $2,3(1-5,2)$ & 0,076 \\
\hline Laktat $5^{\text {th }}$ day & 2,2 & $2,0(0,3-4,5)$ & 2,5 & $2,3(0,3-10,6)$ & 0,286 \\
\hline CRP $1^{\text {th }}$ day & 8,9 & $8,6(0,5-35,4)$ & 12,9 & $8,9(1,3-44,8)$ & 0,311 \\
\hline CRP 5 ${ }^{\text {th }}$ day & 7,1 & $2,8(0,3-47,8)$ & 10,7 & $8,7(0,7-41,7)$ & $\mathbf{0 , 0 1 7}$ \\
\hline PCT $1^{\text {th }}$ day & 8,5 & $1,2(0,0-44)$ & 5,2 & $1,1(0,0-35,6)$ & 0,881 \\
\hline PCT $5^{\text {th }}$ day & 1,7 & $0,3(0,0-20,9)$ & 4,1 & $0,8(0,0-30,7)$ & $\mathbf{0 , 0 3 5}$ \\
\hline SAPS II & 38,1 & $35(19-70)$ & 48,7 & $46(34-69)$ & $<0,001$ \\
\hline $\begin{array}{l}\text { SAPS II predictive mortality } \\
\text { percentage }(\%)\end{array}$ & 26,1 & $17(6-82)$ & 43,1 & $39(17-81)$ & $<0,001$ \\
\hline
\end{tabular}

